\title{
Cardiac contractile function and mitochondrial respiration in diabetes-related mouse models
}

\author{
Camille Marciniak, Xavier Marechal, David Montaigne, Remi Neviere and Steve Lancel
}

\begin{abstract}
Background: Pathophysiological processes underlying diabetic-related cardiomyopathies are complex. Mitochondria dysfunction is often described as a cause of cardiac impairment but its extent may depend on the type of experimental diabetes. Here we proposed to compare drug- or diet-induced models of diabetes in terms of metabolic features, cardiac and mitochondrial functions.

Methods: Mice were fed with regular chow or fat-enriched diet. After three weeks, they received either citrate or streptozotocin injections for five consecutive days. Metabolic parameters, myocardial contractile function and mitochondrial respiration were measured after three more weeks. Fat mass volumes were assessed by magnetic resonance imaging. Oral glucose tolerance test, insulin tolerance test, triglyceride and adipocytokine quantification were evaluated to establish metabolic profiles. Cardiac function was assessed ex vivo onto a Langendorff column. Isolated cardiac mitochondria respiration was obtained using high-resolution oxygraphy.

Results: Mice fed with the fat-enriched regimen presented abdominal obesity, increased blood glucose, elevated leptin level, glucose intolerance, and insulin resistance. Mice treated with streptozotocin, independently of the regimen, lost their capacity to release insulin in response to glucose ingestion. Mice fed with regular chow diet and injected with streptozotocin developed cardiac dysfunction without mitochondrial respiration defect. However, both groups of high-fat diet fed mice developed cardiac alterations associated with reduction in mitochondrial oxygen consumption, despite an increase in mitochondrial biogenesis signalling.
\end{abstract}

Conclusions: We explored three animal models mimicking type 1 and 2 diabetes. While cardiac dysfunction was present in the three groups of mice, mitochondrial respiration impairment was only obvious in models reproducing features of type 2 diabetes.

Keywords: Metabolic syndrome, Diabetes, Heart, Mitochondria, Respiration, Streptozotocin, High-fat diet

According to the American Diabetes Association, diabetes affects more than 25 million people in the United States and will impinge 366 million people in the world in 2030 [1]. Despite established compounds or drugs in development [2] and surgical interventions [3], human and economical costs remain enormous. Development of new experimental models reproducing diabetes mellitus and its complications is indispensable to have a deeper understanding of the pathophysiology and to develop innovative therapeutics.

Several species are used to reproduce diabetes such as nonhuman primates [4] or pigs [5]. The most frequently

\footnotetext{
* Correspondence: steve.lancel@univ-lille2.fr

EA 4484 - Physiology Department, Faculty of Medicine, Lille 2 University, 1, place de Verdun, Lille 59045, France
}

used animals are rodents, especially mice, because of their cost, size and availability. On the first hand, spontaneous monogenic modified mice such as $\mathrm{db} / \mathrm{db}$ and $\mathrm{ob} / \mathrm{ob}$ mice $[6,7]$ as well as animals fed with high-fat diet have been developed. They share common features of type 2 diabetes, such as glucose intolerance, insulin resistance and dyslipidemia. On the other hand, OVE26 and Akita mice or injection of the toxic pancreatic agent streptozotocin (STZ) have been documented to reproduce characteristics of type 1 diabetes [8-10]. In both cases, cardiovascular dysfunction has been described either in basal conditions or under biological stress like $\beta$-adrenergic stimulation [11].

Among the numerous hypotheses advanced to explain diabetes-related cardiomyopathy, including calcium 
mishandling, sarcomere disruption or oxidative stress [12], mitochondrial dysfunction has been described as one of the most important. Indeed, mitochondria occupy approximately $30 \%$ of the heart volume [13] and these organelles are constantly solicited to produce ATP, which is required for cardiac contraction. It is thus obvious that defects in mitochondrial population quality may impair cardiac function. Many murine models of type 2 diabetes, including mice with genetic modifications, fed with high-fat diet associated or not with low doses of STZ, extensively described an association between cardiac dysfunction and mitochondria alterations, including reduced ATP production, defects in mitochondrial respiration despite stimulation of biogenesis signalling and modified proteome [14-16]. Such observations have also been obtained in the human heart from type 2 diabetic patients [17]. Surprisingly, cardiac mitochondrial dysfunction in models of type 1 diabetes is less characterized, differs from type 2 diabetes and from a model to another $[10,18,19]$. Finally, because of the variety of protocols published in distinct papers, it is not clear whether the intensity of mitochondrial dysfunction is correlated with the severity of cardiac dysfunction and whether mitochondrial impairment occurs at the same time in both types of diabetes.

In this study, we evaluated cardiac function and respiration of heart mitochondria in three different models of diabetes. First, we compared metabolic parameters such as glucose tolerance or insulin resistance in mice fed with regular chow diet and injected with STZ, mice fed with high-fat diet treated with citrate buffer or STZ to mice fed with normal diet injected with citrate buffer. Then, we measured myocardial contractile function and mitochondrial respiration in order to determine whether mitochondrial alterations precede diabetic-related cardiomyopathy.

\section{Methods}

\section{Experimental models}

Five-week old female C57/BL6J mice (Charles River, L'Arbresle, France) were randomly separated in two groups: the first one had free access to normal chow diet (ND), the other one to high-fat diet (HFD, D12492, SSNIFF, Soest, Germany). Food composition and energy sources are provided Table 1 . Then, both groups of mice received either streptozotocin $(60 \mathrm{mg} / \mathrm{kg}$ intraperitoneally, 1 injection per day for five consecutive days, Sigma Aldrich, St Quentin Fallavier, France), or the equivalent volume of citrate buffer that was used to dissolve streptozotocin $(0.1 \mathrm{M}, \mathrm{pH} 4.5,1$ injection per day for five days). After injections, mice were housed for three more weeks and fed ad libitum with their respective food. Thus, after six weeks of feeding, four groups were obtained: ND/CITRATE, ND/STZ, HFD/CITRATE and HFD/STZ. There were at least two full days between tests to avoid potential stress confounding effects. All
Table 1 Nutrient composition and energy sources of normal and high-fat diets

\begin{tabular}{lll}
\hline & Normal diet & High-fat diet \\
\hline Crude protein (\%) & 16.4 & 24.1 \\
Fat (\%) & 4 & 34.6 \\
Carbohydrate (\%) & 48.5 & 25.3 \\
Fiber (\%) & 18.5 & 6 \\
Ash (\%) & 4.9 & 6 \\
Energy density (kJ/g) & 12.6 & 24 \\
Calories from proteins (\%) & 22 & 19 \\
Calories from fat (\%) & 12 & 60 \\
Calories from carbohydrates (\%) & 66 & 21 \\
\hline
\end{tabular}

experimental procedures were approved by our institutional guidelines (DDSV Permit Number 59-350206) and were conducted according to NIH instructions.

\section{Magnetic resonance imaging of fat distribution}

Once anesthetized by inhaling isoflurane, mice were imaged on a Biospec 7-Tesla magnetic resonance imaging unit (Biospec, BrukerBioSpin SA, Wissembourg, France). Sequences were acquired in the abdomen, around the kidney region. The T1 spin-echo images were obtained with a thickness of $1 \mathrm{~mm}$, a repetition time of $200 \mathrm{~ms}$ and an echo time of $27 \mathrm{~ms}$. Data were analyzed with OsiriX software. Subcutaneous and abdominal fat mass volumes (expressed in $\mathrm{cm}^{3}$ ) were determined manually.

\section{Oral glucose tolerance test OGTT}

In order to evaluate glucose tolerance, mice were subjected to a 12-h fasting period. Then, they were forcefed with a glucose solution ( $2 \mathrm{mg} / \mathrm{g}$ total body weight) in approximately $200 \mu \mathrm{L}$ of water. One drop of blood obtained from a tail incision was used to determine glucose concentration by the use of a glucose meter. Measurements were obtained before ingestion and 10, 20, 30, 60 and 120 min after glucose challenge.

\section{Insulin tolerance test ITT}

Fasted mice received intraperitoneally fast insulin $(0.5 \mathrm{mU} / \mathrm{g}$ total body weight in saline solution). Blood glucose concentration was measured before and 15, 30 and $60 \mathrm{~min}$ after the injection.

\section{Blood collection and analysis of plasma}

After fasting, tail incision was made and blood $(100 \mu \mathrm{L})$ was collected into EDTA-coated tubes. Sample was spun at $1,000 \mathrm{~g}$ for $15 \mathrm{~min}$ and plasma was aliquoted and stored at $-80^{\circ} \mathrm{C}$ until use. Plasmatic adiponectin, leptin, TNF- $\alpha$, IL- 6 and MCP1 were quantified with multiplex immunoassay kits (Millipore, Molsheim, France). True triglycerides were measured with the determination kit 
from Sigma Aldrich (St Quentin Fallavier, France), according to manufacturer's instructions.

\section{Insulin response to glucose challenge}

Blood samples $(30 \mu \mathrm{L})$ were collected from fasted mice. Then, animals were force-fed with a glucose solution ( $2 \mathrm{mg} / \mathrm{g}$ total body weight). Ten minutes later, $30 \mu \mathrm{L}$ of blood were withdrawn for plasma preparation. Then, insulin was measured with the insulin (Mouse) EIA kit (Alpco, Salem, NH, USA) according to manufacturer's instructions.

\section{Pancreas immunohistochemistry}

After mouse euthanasia, pancreas was dissected, fixed in Bouin solution and embedded in paraffin. Then, sections of $6 \mu \mathrm{m}$ thickness were prepared and put onto superfrost slides. After paraffin removal, tissue was rehydrated in solutions of decreasing alcohol concentration. Slides were immersed into citrate buffer $(0.01 \mathrm{M}, \mathrm{pH} \mathrm{6})$ in order to unmask antigens. Then, endogenous peroxidase activity was blocked by incubating sections in $3 \% \mathrm{H}_{2} \mathrm{O}_{2}$ diluted in methanol. After incubation in PBS $+3 \%$ bovine serum albumin, monoclonal anti-insulin antibody (1/100, AbD Serotec Colmar, France) was added to the sections and incubated at $4^{\circ} \mathrm{C}$ overnight. After rinsing, peroxidaseconjugated anti-mouse antibody was incubated for $1 \mathrm{~h}$ at room temperature. 3,3'-diaminobenzidine (Bethyl Laboratories, Inc., Montgomery, TX, USA) was applied for $15 \mathrm{~min}$ at room temperature. Slides were counterstained by immersion in hematoxylin. After rinsing, slides were mounted and observed under microscope with $\times 100$ and $\times 400$ magnification.

\section{Isolated and perfused heart}

After cervical dislocation, heart was excised and aorta was cannulated onto a Langendorff column. Heart was perfused in a retrograde manner with oxygenated $\mathrm{O}_{2} 95 \%$, $\mathrm{CO}_{2} 5 \%$ ) modified Krebs-Henseleit (consisting in $\mathrm{NaCl}$ $120 \mathrm{mM}, \mathrm{KCl} 4.8 \mathrm{mM}, \mathrm{KH}_{2} \mathrm{PO}_{4} 1.2 \mathrm{mM}, \mathrm{MgSO}_{4} 1.2 \mathrm{mM}$, $\mathrm{NaHCO}_{3} 25 \mathrm{mM}, \mathrm{CaCl}_{2} 1.25 \mathrm{mM}$, glucose $\left.11 \mathrm{mM}\right)$ at $37^{\circ} \mathrm{C}$ with a constant perfusion rate $(2.5 \mathrm{~mL} / \mathrm{min})$. In some experiments, $0.8 \mu \mathrm{g} / \mathrm{mL}$ isoprenaline was added in the buffer. A metal hook was inserted into the apex. The hook was connected to a calibrated dynamometer, which was connected to a pre-amplifier linked to the acquisition station (Powerlab, ADInstrument, Oxford, United Kingdom). Heart spontaneously beat with a 2 g preload. Developed force and its first derivatives were measured in absence or presence of isoprenaline. Heart work was defined as the product of force $\mathrm{x}$ heart frequency.

\section{Mitochondria isolation and function}

Cardiac mitochondria were isolated as previously described [20]. Mitochondria $(200 \mu \mathrm{g})$ were introduced into O2K oxygraph chambers (Oroboros Instruments, Innsbruck, Austria) to evaluate their oxygen consumption in presence of different compounds. In a first series of experiments, pyruvate $(5 \mathrm{mM})$, glutamate $(5 \mathrm{mM})$ and mala $(2 \mathrm{mM})$ were added. After signal stabilization, ADP $(0.5 \mathrm{mM})$ was injected in order to measure oxygen consumption when oxidative phosphorylation occurs. The second series of experiments consisted in adding palmitoylcarnitine $(20 \mu \mathrm{M})$ in presence of malate $(2 \mathrm{mM})$. Then ADP $(0.5 \mathrm{mM})$ was injected into the chambers in order to evaluate respiration with substrates subjected to $\beta$-oxidation.

\section{Mitochondrial DNA copy number}

Total DNAs were extracted with QIAamp DNA mini kit (Qiagen, Courtaboeuf, France) according to manufacturer's instructions. Nucleic acid concentration and purity were measured at 260 and $280 \mathrm{~nm}$ with the Nanodrop reader (Nanodrop products, Wilmington, DE, USA). Then $40 \mathrm{ng}$ of DNA were used to perform quantitative PCR with FastStart Universal SYBR Green (Roche Applied Science, Meylan, France). The following primers were utilized: mtCOII Forward AACCATAGGGCACCAAT GATAC, Reverse GGATGGCATCAGTTTTAAGTCC [GeneID: 17709]; PPIA Forward ACACGCCATAATG GCACTGG, Reverse CAGTCTTGGCAGTGCAGAT [GeneID: 268373].

\section{RTqPCR}

Total RNAs were extracted from 20 mg heart tissue with $1 \mathrm{~mL}$ Trizol reagent and purified with PureLink Microto-Midi kit (Life Technologies SAS, Saint Aubin, France). DNA was systematically removed by DNase treatment. Then, reverse transcription with random hexamers and Transcriptor First Strand cDNA Synthesis kit (Roche Applied Science) was performed according to manufacturer's instructions. Amplification was done with FastStart Universal SYBR Green and the following primers: PGC- $1 \alpha$ Forward CGGAAATCATATCCAACCAG, Reverse TGAG GACCGCTAGCAAGTTTG [NM_008904], 18S Forward CGGCGACGACCCATTCGAAC, Reverse GAATCGAAC CCTGATTCCCCGTC [NR_003278].

\section{Statistical analysis}

Presented data are means \pm SEM. Analysis was performed with GraphPad Prism software 5.0 (San Diego, CA, USA). Statistical analysis was carried out using twotailed unpaired $t$-test when comparing two groups and one-way ANOVA followed by Bonferroni post-hoc tests when comparing more than three groups. Two-way ANOVA was performed to determine time influence on the measured parameters and was used for analysis of food intake, calorie intake, variation of weight, OGTT and ITT. 


\section{Results}

Food intake, weight gain and fat distribution

Prior to cardiovascular and mitochondrial evaluations, we compared metabolic phenotypes of ND/STZ, HFD/ CITRATE and HFD/STZ to ND/CITRATE. Three weeks after the beginning of the regimen, mice fed with HFD started eating less than their littermates under ND (Figure 1A). Despite this difference, cumulative energy intake in the HFD mice was significantly higher than in the ND animals from the first to the last week of specific diet (Figure 1B). Mouse weight increased faster in HFD groups than in the ND ones (Figure $1 C$ ). Injections of STZ (60 $\mathrm{mg} / \mathrm{kg}$ total body weight) did not modify the amount of ingested food as ND/STZ and HFD/STZ ate as much as ND/CITRATE and HFD/CITRATE, respectively (Figures 1A and B). Similarly, body weights between
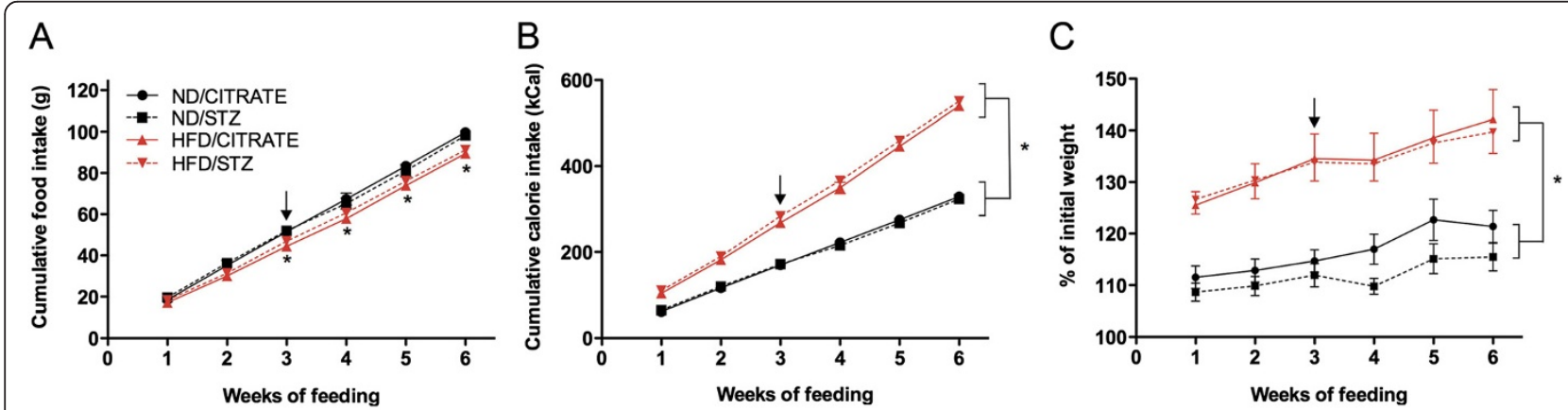

D
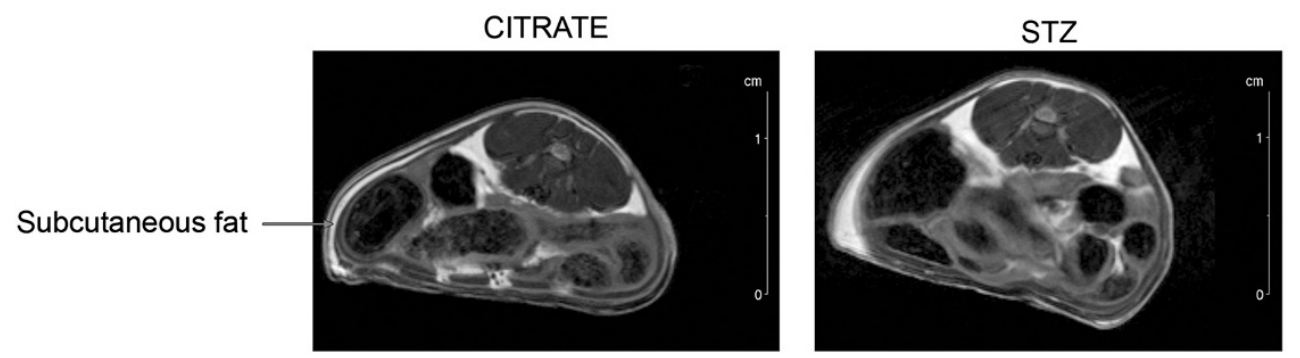

ND
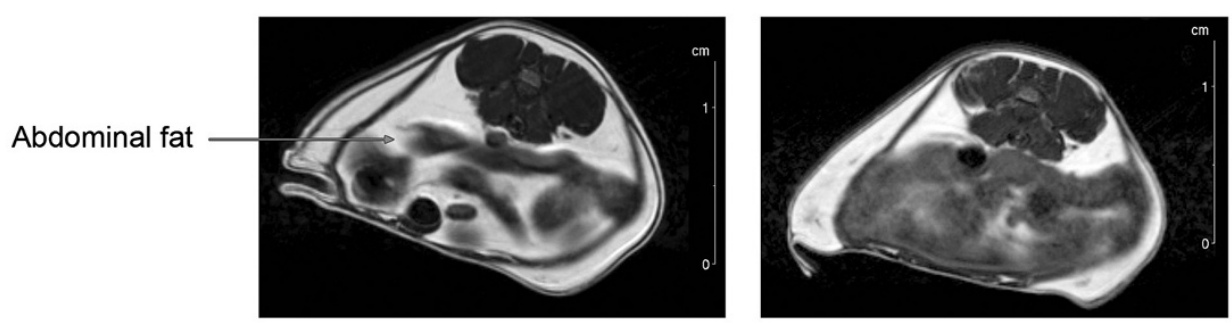

HFD
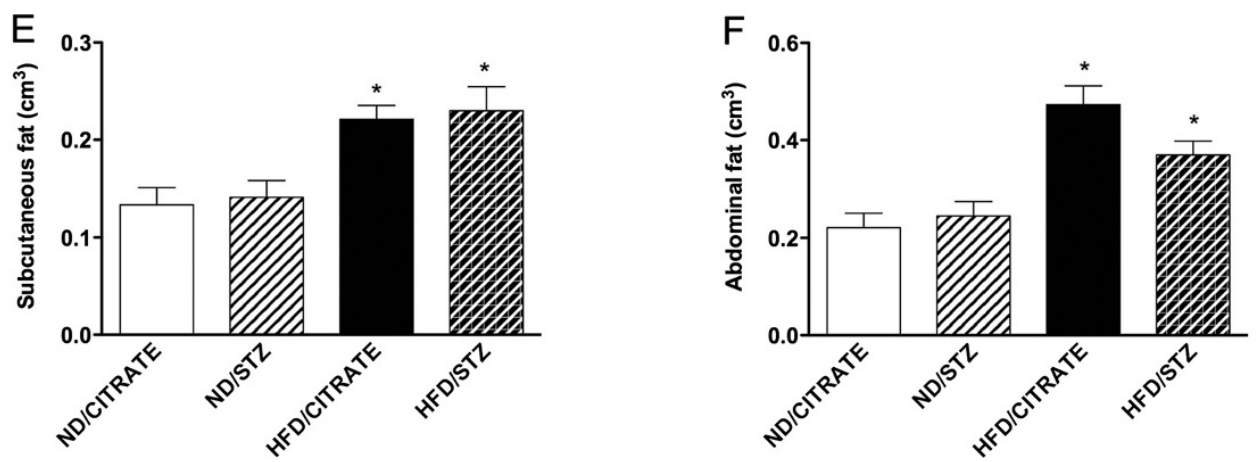

Figure 1 Food intake, weight gain and fat volume in the four groups of mice. (A) Cumulative food intake and (B) cumulative calorie intake over weeks of feeding. (C) Gain of weight compared to initial weight. Circle: ND/CITRATE, square: ND/STZ, triangle: HFD/CITRATE, diamond: HFD/ STZ mice. Arrows indicate when streptozotocin (STZ) or citrate buffer were injected. (D) MRI images of abdominal region in ND/CITRATE, ND/STZ, HFD/CITRATE, HFD/STZ mice fed for 6 weeks. Arrows indicate subcutaneous and abdominal fat. Volume quantification of (E) subcutaneous and (F) abdominal fat. $\mathrm{n}=8$ per group, ${ }^{*} \mathrm{p}<0.05$ vs. ND/CITRATE mice. 
citrate- or STZ-injected mice within ND or HFD groups were not significantly different (Figure 1C).

To further characterize their phenotypes, mice were subjected to MRI to quantify fat mass. As depicted in Figure 1D, fat distribution between citrate- and STZinjected ND mice was similar. These results were confirmed by image quantification (Figures $1 \mathrm{E}$ and $\mathrm{F}$ ). In both groups of HFD mice, hypersignal was stronger in subcutaneous and abdominal compartments (Figure 1D). Analysis revealed that, compared to ND/CITRATE, subcutaneous fat volume increased by $77 \%$ while abdominal fat volume doubled (Figures $1 \mathrm{E}$ and $\mathrm{F}$ ) in both HFD groups. STZ injections had no effects on fat deposition neither in ND nor in HFD mice (Figures 1D-F).

\section{Glucose intolerance}

OGTT was performed to evaluate glucose tolerance in the four different groups either after one week of feeding (no injections), three weeks (just before injections of citrate or STZ), or six weeks, i.e. three weeks after the first injections. As presented Figure 2A, mice had similar response to glucose ingestion after one week of HFD with no injections. After three weeks of feeding (Figure 2B), there were no significant differences between ND mice that will subsequently be injected with citrate buffer (ND/CITRATE) or STZ (ND/STZ). Similar results were obtained within the HFD groups (Figure 2B). Nevertheless, as soon as three weeks under specific regimens, both groups of mice fed with HFD started showing glucose intolerance, as their blood glucose concentration remained elevated $60 \mathrm{~min}$ after ingestion compared to ND (Figure 2B). Finally, OGTT was performed three weeks after citrate or STZ injections (Figure 2C). Compared to ND/CITRATE mice, ND/STZ and mice fed with HFD that received or not STZ had higher glucose concentration 20, 30 and $60 \mathrm{~min}$ after glucose forcefeeding. Plus, after two hours, glucose concentration remained above $200 \mathrm{mg} / \mathrm{dL}$ in the HFD/STZ group (Figure 2C). All subsequent experiments were performed six weeks after the onset of the regimen.

\section{Insulin resistance and secretion capacity}

In HFD and HFD/STZ groups, slope of glucose concentration reduction after insulin injection was reduced compared to ND/CITRATE mice (Figure 3A). In addition, after $60 \mathrm{~min}$, blood glucose concentration was reduced by only $20 \%$ in HFD-STZ and HFD/CITRATE while it decreased by $50 \%$ in both ND/CITRATE and ND/STZ (Figure 3A). ND/STZ mice had significant higher glucose concentrations 15 and 30 min after insulin injection.

We then evaluated glucose-stimulated insulin release by measuring circulating insulin before and after an oral glucose challenge. As illustrated in Figure 3B, insulin levels
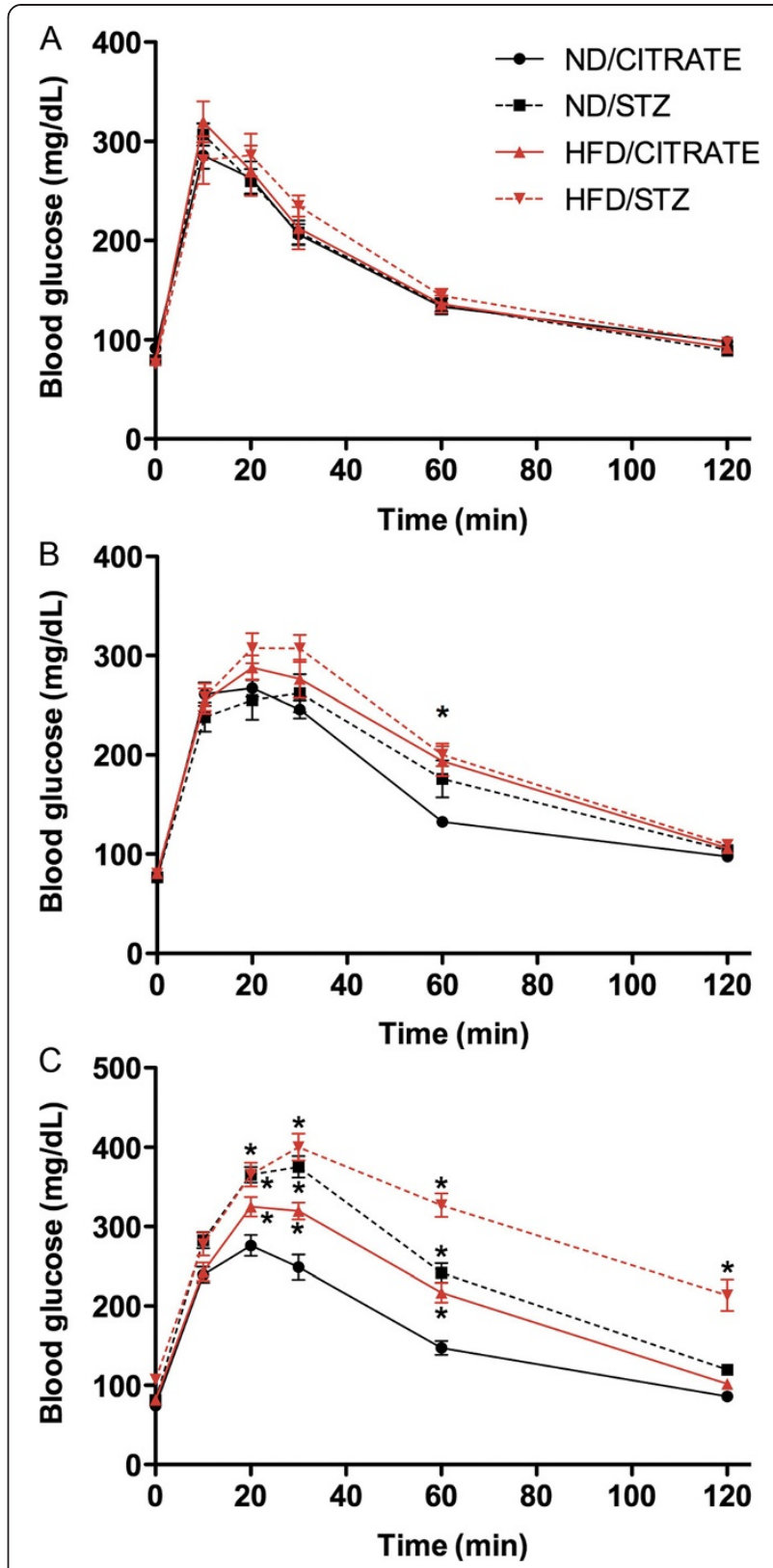

Figure 2 Oral glucose tolerance tests of mice fed for one, three and six weeks. After glucose ingestion, blood glucose concentration was measured at 0, 10, 20, 30, 60, 120 min later in mice fed for one (A), three (B) or six (C) weeks. Circle: ND/CITRATE, square: ND/STZ, triangle: HFD/CITRATE, diamond: HFD/STZ mice. $n=16$ per group, ${ }^{*} \mathrm{p}<0.05$ vs. ND/CITRATE.

underwent a 11-fold increase either in ND/CITRATE or HFD/CITRATE mice. Mice treated with STZ lost their capacity to respond to glucose as insulin levels barely increased by 3 (Figure 3B). Moreover HFD/CITRATE mice significantly released more insulin than ND/CITRATE littermates (Figure 3B).

Finally, we qualitatively evaluated whether such alterations could be attributed to reduced size of pancreatic 

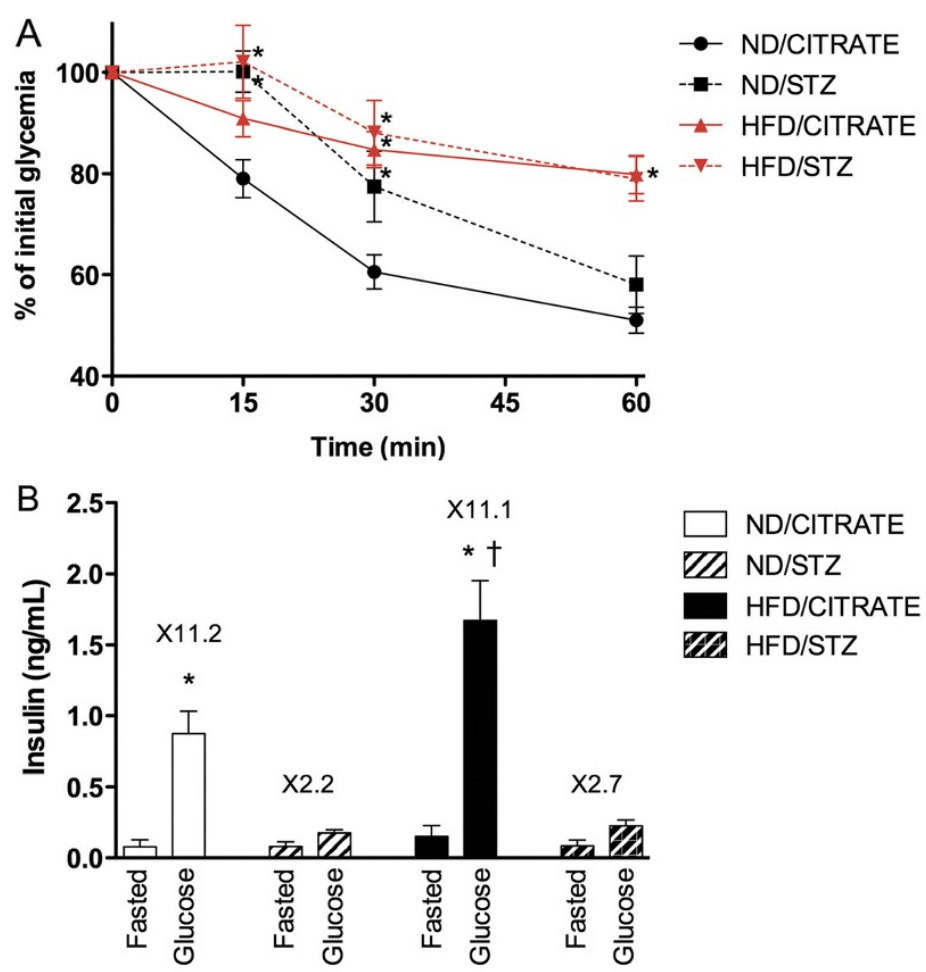

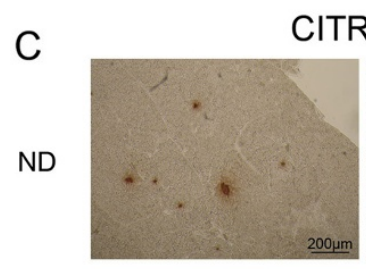

$\mathrm{X} 100$

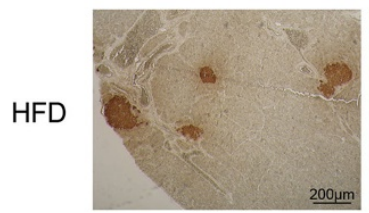

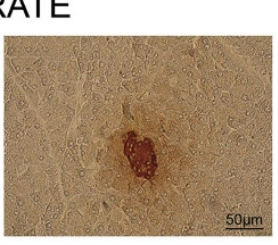

X400

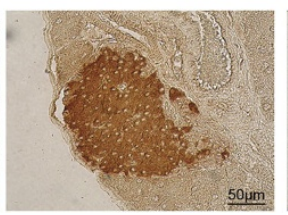

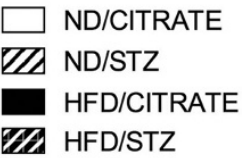

IIA HFD/STZ

Figure 3 Streptozotocin and high-fat diet induced insulin abnormalities after six weeks of feeding. (A) Insulin tolerance test. Blood glucose was measured 15, 30 and 60 min after insulin injection. $n=10,{ }^{*} p<0.05$ vs. ND/CITRATE. (B) Insulin secretion in response of glucose ingestion. $n=5$, ${ }^{*} p<0.05$ compared to fasted conditions, $+p<0.05$ HFD/CITRATE vs. ND/CITRATE. (C) Immunohistochemistry of pancreas using an anti-insulin antibody. Arrows indicate cells with lower staining intensity. Magnifications were $\times 100$ and ×400, as indicated.

islets and insulin content, as STZ is a known-pancreatic poison targeting $\beta$-cells. Islet staining intensity seemed reduced in ND/STZ and HFD/STZ compared to ND/ CITRATE mice (Figure 3C). In HFD/CITRATE group, islet size appeared bigger than in ND/CITRATE group but the staining intensity was not affected (Figure 3C). As expected, size of islets was reduced in both groups treated with STZ (Figure 3C).

\section{Metabolic parameters}

Blood glucose concentration in fasted animals was significantly higher, although moderate, in ND/STZ,
HFD/CITRATE and HFD/STZ as compared to ND/ CITRATE (Table 2). Triglyceride and leptin levels mildly augmented in mice fed with HFD, independently of STZ treatment (Table 2). Inversely, compared to ND/CITRATE, adiponectin level was divided by 2 in HFD/CITRATE group while it diminished by $30 \%$ in HFD/STZ animals (Table 2). $\mathrm{ND} / \mathrm{STZ}$ mice had equivalent amount of adiponectin compared to ND/CITRATE mice (Table 2). Regarding the proinflammatory status, neither MCP-1 (not detectable), nor TNF- $\alpha$, nor IL- 6 was significantly increased although a trend could be observed in ND/STZ, HFD/CITRATE and HFD/STZ compared to ND/CITRATE animals (Table 2). 
Table 2 Blood parameters after six weeks of diet

\begin{tabular}{lllll}
\hline & ND/CITRATE & ND/STZ & HFD/CITRATE & HFD/STZ \\
\hline Glucose (mg/dL) & $74 \pm 2$ & $84 \pm 3^{*}$ & $83 \pm 2^{*}$ & $112 \pm 6^{*}$ \\
$\begin{array}{l}\text { True triglycerides } \\
\text { (mg/dL) }\end{array}$ & $52 \pm 4$ & $60 \pm 10$ & $90 \pm 8^{*}$ & $103 \pm 11 *$ \\
Leptin (ng/mL) & $1.0 \pm 0.2$ & $1.1 \pm 0.1$ & $1.9 \pm 0.2 *$ & $2.3 \pm 0.3 *$ \\
$\begin{array}{l}\text { Adiponectin } \\
\text { ( } \mu \mathrm{g} / \mathrm{mL})\end{array}$ & $30.3 \pm 3.1$ & $30.3 \pm 6.1$ & $15.6 \pm 0.9 *$ & $21.4 \pm 1.5 *$ \\
TNF-a $(\mathrm{pg} / \mathrm{mL})$ & $3.8 \pm 0.5$ & $6.0 \pm 1.2$ & $5.4 \pm 0.8$ & $4.5 \pm 0.4$ \\
IL-6 $(\mathrm{pg} / \mathrm{mL})$ & $7.5 \pm 0.9$ & $12.4 \pm 2.0$ & $14.7 \pm 1.8$ & $13.0 \pm 2.8$ \\
\hline
\end{tabular}

Data are means \pm SEM. $n=8-10$ per group, ${ }^{*} p<0.05$.

\section{Cardiac function}

Isolated heart function was assessed onto a Langendorff apparatus. Under basal conditions, heart work (Figure 4A) and first derivatives $+\mathrm{d} F / \mathrm{d} t$ and $-\mathrm{d} F / \mathrm{d} t$ (Figure 4B), index of contractility and relaxation respectively, were reduced in ND/STZ, HFD/CITRATE and HFD/STZ compared to $\mathrm{ND} / \mathrm{CITRATE}$. In presence of isoprenaline, a $\beta$-adrenergic receptor agonist, both ND/CITRATE and HFD/CITRATE hearts increased cardiac work (Figure 4A) and first derivatives (Figure 4B). In contrast, ND/STZ and HFD/STZ hearts did not significantly respond to $\beta$-adrenergic stimulation (Figure 4).

\section{Mitochondrial respiration and biogenesis}

Mitochondria were isolated from heart and analyzed with an oxygraph. When mitochondria were incubated in presence of palmitoylcarnitine and malate, respiration rate was similar in the four groups. Addition of ADP in the respiration media led to a 6.8- and 6.1-fold increase in oxygen consumption in the ND/CITRATE and ND/ STZ mice, respectively (Figure 5A). This increase in respiration was significantly lower in HFD/CITRATE and HFD/STZ mice (Figure 5A). Similarly, no differences between groups were observed in presence of glutamate/ malate alone (Figure 5B). Activation of oxidative phosphorylation by ADP resulted in a 5-fold increase in ND/ CITRATE and ND/STZ whereas it increased by 3 in the HFD/CITRATE and HFD/STZ (Figure 5B). These mitochondrial alterations observed in HFD/CITRATE and HFD/STZ were associated with an increase in mitochondrial biogenesis signalling. Indeed, compared to ND/ CITRATE, mtDNA copy number was higher in HFD/ CITRATE and HFD/STZ (Figure 5C). Similar results were obtained for PGC-1 $\alpha$ mRNA expression (Figure 5D).

\section{Discussion}

The aim of this study was to characterize the metabolic phenotype of three diabetes-related animal models induced by HFD and the pancreatic poison STZ and to evaluate whether cardiac dysfunction was always associated with mitochondrial respiration impairment.

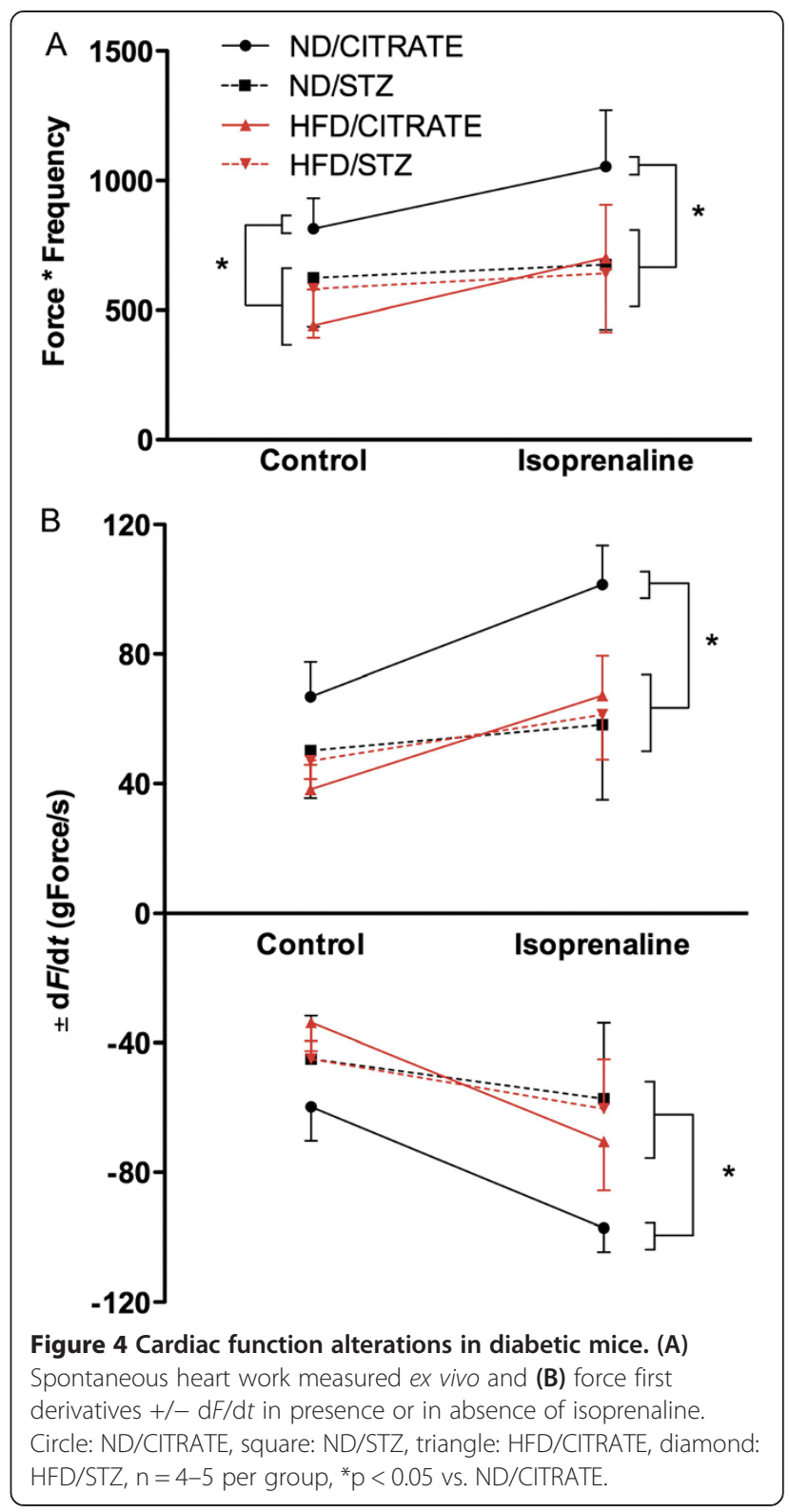

As a prerequisite before assessment of myocardial and mitochondrial functions, we evaluated metabolic features of the three diabetes-related models obtained in C57BL6/J mice, a strain known to respond to diet-induced obesity and to develop insulin resistance and glucose intolerance [21]. First, HFD mice gained more weight and consequently developed both subcutaneous and abdominal adipose tissues, as assessed by MRI. This was associated with a mild plasma true triglyceride elevation. Visceral adipose tissue, now considered as an endocrine tissue [22], produces pro-inflammatory cytokines such as TNF- $\alpha$ and IL6 [23]. Here, we could not detect significant elevation in these two molecules. This may be attributed to the relatively short-term regimen (6 weeks) or to the C57BL6/J genetic background. Indeed $\mathrm{Balb} / \mathrm{c}$ or $\mathrm{FVB} / \mathrm{N}$ mice are 


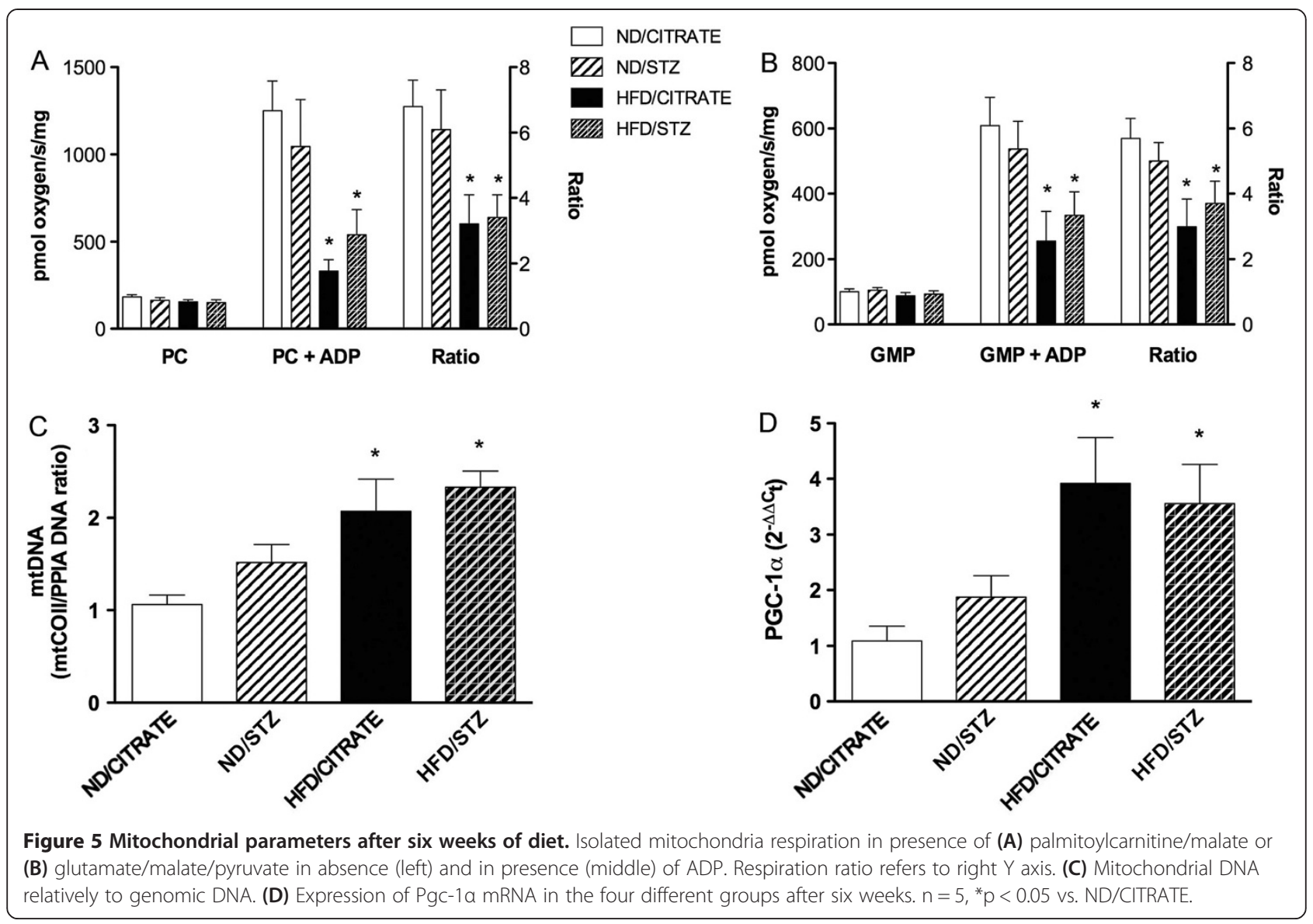

more prone to develop HFD-induced inflammation [21]. Abdominal adipocytes also secrete leptin and adiponectin. As expected, the elevation in visceral fat was associated with increase in leptin circulating levels while adiponectin was reduced. Despite higher leptin concentration in the HFD fed animals, food intake barely reduced and mice kept accumulating fat, consistently with other studies [24]. STZ had no effects on leptin and adiponectin levels.

A deeper analysis of diabetes-related features consisted in studying glucose intolerance and insulin resistance. HFD/CITRATE or HFD/STZ mice had a lower rate of glucose disposal after insulin injection and glucose ingestion, as well as a moderate increase in fasting blood glucose concentration, featuring an insulin resistant status. Several mediators such as leptin, which reduces insulininduced fatty acid oxidation and triacylglycerol synthesis $[25,26]$, or fatty acids, which compete with glucose for their mitochondrial oxidation [27,28], are commonly suggested as contributors of insulin resistance and glucose intolerance. As observed in other experimental studies and in humans [29-31], we also detected bigger pancreatic $\beta$ cell islets and higher plasmatic insulin levels ten minutes after glucose ingestion in HFD/CITRATE mice. This metabolic phenotype indicates that HFD/CITRATE mice manifested features of a pre-diabetic state. We reproduced effects of STZ, an alkylating compound causing beta pancreatic cell death, described by others [32-34]. Indeed, ND/STZ and HFD/STZ mice released less insulin consecutively to glucose stimulation. Taken together, these results suggest that ND/STZ, HFD/CITRATE and HFD/ STZ were more related to type 1 diabetes, pre-diabetes and established type 2 diabetes, respectively.

Diabetes-related cardiovascular alterations have been documented in both type 1 and type 2 rodent models [16,35-38]. Here we aimed to determine whether intrinsic myocardial contractile function was affected in these different settings of diabetes. In order to avoid confounding effects of circulating leptin, triglycerides, cytokines or insulin on the heart, we used isolated and perfused heart preparations onto a Langendorff apparatus. First, isolated hearts from STZ-treated animals did not respond to $\beta$ adrenergic stimulation. This could be related to a reduction in $\beta 1$ and $\beta 2$ adrenoreceptor expression while the $\beta 3$ isoform, mediating negative inotropic effect, may rise [39]. Then, despite the mild elevation in fasting blood glucose and triglyceride concentration and an exploration close to the onset of diabetes, we found a marked intrinsic contractile dysfunction of the myocardium in the three 
diabetes-related models. Increased fatty acid oxidation at the expense of glucose utilization, expansion of connective tissue, oxidative stress, calcium mishandling and reduction in SERCA expression have been proposed to explain diabetic cardiac dysfunction [12,35]. In addition, several studies identified mitochondria as a potential cause of myocardial dysfunction in diabetes. Thanks to the early development of diabetic cardiomyopathy, we decided to explore whether this organ dysfunction was always associated with mitochondrial impairment.

We highlighted a profound mitochondrial respiration reduction in insulin-resistant HFD/CITRATE and HFD/ STZ mice. Consistently studies on cardiac-specific knockout mice for the insulin receptor (CIRKO) demonstrated that lack of insulin signalling in the myocardium leads to impaired contractile function along with mitochondria respiratory defects and ATP synthesis rate reduction that progress with age [40]. In addition, reduction of mitochondrial respiration was associated with stimulation of mitochondrial biogenesis signalling as evidenced by increased PGC- $1 \alpha$ mRNA expression and mtDNA copy number. PGC-1 $\alpha$ is a major transcription factor controlling mitochondrial biogenesis. Genetic deletion of PGC$1 \alpha$ causes reduced cardiac ATP production and the incapacity to respond to a stress such as exercise or adrenergic stimulation $[41,42]$. On the contrary, Lehman et al. [43] showed that, despite PGC- $1 \alpha$-mediated mitochondria number increase, contractile function was severely affected because of sarcomere misalignment. These studies emphasize that mitochondrial function had to be tightly regulated to exert beneficial effects on cardiac function. Mitochondrial population quality also depends on autophagy and dynamics, processes that are also involved in diabetes. For instance, in a severe model of type 1 diabetes, $\mathrm{Xu}$ et al. [44] described that autophagy is reduced in the diabetic heart. Surprisingly, inhibition of autophagy exerted cardioprotective effects, probably through at least in part mitophagy activation. Alterations of mitochondrial dynamics have also been reported in failing diabetic human hearts [45]. Although we did not examined oxidative damage in these hearts, increased in mitochondrial reactive oxygen species production may also contribute to the mitochondrial phenotype. Nakamura et al. [46] demonstrated that, for instance, in $\mathrm{db} / \mathrm{db}$ mice, mitochondrialderived ROS generation due to hyperglycemia and lipid accumulation activated p53 and its target SCO2. As a consequence, higher expression of $\mathrm{SCO} 2$ resulted in increases in fatty acid uptake, mitochondrial oxygen consumption and ROS production.

In the present study, we did not observe defects in mitochondrial respiration in ND/STZ mice, neither with pyruvate/glutamate nor with palmitoylcarnitine as substrates, while cardiac dysfunction was patent, suggesting that defects in mitochondrial respiration per se may not be the primary cause of cardiac dysfunction. While studies described similar results in rats [47], others reported that palmitoylcarnitine-dependent mitochondrial respiration could remain identical between control and STZ-injected despite a reduction in ATP production rate [48]. More recently, in a chronic model of type 1 diabetes, Vadvalkar et al. [49] demonstrated that heart mitochondria may have substrate inflexibility as their respiration was reduced only with non-fatty acid substrates. They also showed that protein lysine acetylation reproduced inhibition of non-fatty acid oxidation by mitochondria. The authors thus hypothesized that discrete mitochondrial changes such as protein lysine acetylation could impact oxidative phosphorylation before the onset of type 1 diabetic cardiomyopathy. However, in our study, we could not detect mitochondrial impairment with pyruvate/glutamate. This may be explained by the early investigation after type1-like diabetes induction as well as the mild phenotype we observed in terms of hyperglycemia and dyslipidemia. This model may not recapitulate the whole pathogenesis of a more severe and chronic type 1 diabetes. Finally, other mitochondrial functions may be affected during diabetes. Indeed a recent report [50] identified that ATP-dependent potassium channel expression is diminished by type 1 diabetes. As a consequence, diazoxide-induced depolarization was lower in diabetic mitochondria. Interestingly, interfibrillar mitochondria were more sensitive to diazoxide than subsarcolemmal mitochondria. This result underlines that mitochondrial populations may be differently affected by diabetes.

In conclusion, mechanisms responsible for the diabeticrelated cardiomyopathies depend on duration, type and severity of diabetes. Injections of streptozotocin in mice fed with regular chow diet led to a type-1 like diabetic mouse model that displayed cardiovascular alterations without mitochondrial respiration impairment. On the contrary, cardiac dysfunction of mice fed with HFD that received or not STZ was associated with a dramatic reduction in mitochondrial respiration despite mitochondrial biogenesis activation.

\section{Abbreviations}

ADP: Adenosine diphosphate; ANOVA: Analysis of variance; DNA: Deoxyribonucleic acid; EDTA: Ethylenediaminetetraacetic acid; EIA: Enzyme immunoassay; HFD: High-fat diet; IL-6: Interleukin-6; ITT: Insulin tolerance test; MCP1: Monocyte chemotactic protein-1; MRI: Magnetic resonance imaging; ND: Normal diet; NIH: National Institutes of Health; OGTT: Oral glucose tolerance test; PBS: Phosphate-buffered saline; PCR: Polymerase chain reaction; PGC-1a: Peroxisome proliferator-activated receptor gamma coactivator 1; SEM: Standard error of the mean; STZ: Streptozotocin; TNF-a: Tumor necrosis factor-a.

\section{Competing interests}

The authors declare that they have no competing interests.

\section{Author contributions}

CM conducted the experiments and contributed to study implementation, analysis, data interpretation and manuscript writing. XM and DM performed analyzed and interpreted myocardial function experiments. RN participated to study design, analysis and interpretation, revised the manuscript. SL designed 
the study, contributed to study implementation, analysis, data interpretation and wrote the manuscript. All authors read and approved the final manuscript.

\section{Acknowledgments}

We thank Florent Auger-IMPRT114 for his technical assistance in 7-tesla MRI, Bernadette Lescure-IMPRT65 for adipocytokine determination. This work was supported by EA4484 - Université Lille2 - Ministère de l'Enseignement Supérieur et de la Recherche; CPER "cardiodiabète 2008" - FEDER Région Nord Pas-de-Calais 08480265; Fondation de France 2009002501 and Fondation Coeur et Artères FCA09T6.

Received: 26 March 2014 Accepted: 23 July 2014

Published: 21 August 2014

\section{References}

1. Wild S, Roglic G, Green A, Sicree R, King H: Global prevalence of diabetes: estimates for the year 2000 and projections for 2030. Diabetes Care 2004, 27:1047-1053.

2. Verspohl EJ: Novel pharmacological approaches to the treatment of type 2 diabetes. Pharmacol Rev 2012, 64:188-237

3. Dixon JB, le Roux CW, Rubino F, Zimmet P: Bariatric surgery for type 2 diabetes. Lancet 2012, 379:2300-2311.

4. de Koning EJ, Bodkin NL, Hansen BC, Clark A: Diabetes mellitus in macaca mulatta monkeys is characterised by islet amyloidosis and reduction in beta-cell population. Diabetologia 1993, 36:378-384.

5. Xi S, Yin W, Wang Z, Kusunoki M, Lian X, Koike T, Fan J, Zhang Q: A minipig model of high-fat/high-sucrose diet-induced diabetes and atherosclerosis. Int J Exp Pathol 2004, 85:223-231.

6. Hummel KP, Dickie MM, Coleman DL: Diabetes, a new mutation in the mouse. Science 1966, 153:1127-1128.

7. Pelleymounter MA, Cullen MJ, Baker MB, Hecht R, Winters D, Boone T, Collins F: Effects of the obese gene product on body weight regulation in ob/ob mice. Science 1995, 269:540-543.

8. Parker G, Taylor R, Jones D, McClain D: Hyperglycemia and inhibition of glycogen synthase in streptozotocin-treated mice: role of O-linked N-acetylglucosamine. J Biol Chem 2004, 279:20636-20642.

9. Liang Q, Carlson EC, Donthi RV, Kralik PM, Shen X, Epstein PN: Overexpression of metallothionein reduces diabetic cardiomyopathy. Diabetes 2002, 51:174-181.

10. Bugger H, Boudina S, Hu XX, Tuinei J, Zaha VG, Theobald HA, Yun UJ, McQueen AP, Wayment B, Litwin SE, Abel ED: Type 1 diabetic akita mouse hearts are insulin sensitive but manifest structurally abnormal mitochondria that remain coupled despite increased uncoupling protein 3. Diabetes 2008, 57:2924-2932.

11. Boudina S, Abel ED: Diabetic cardiomyopathy revisited. Circulation 2007, 115:3213-3223.

12. Boudina S, Abel ED: Diabetic cardiomyopathy, causes and effects. Rev Endocr Metab Disord 2010, 11:31-39.

13. Schaper J, Meiser E, Stämmler G: Ultrastructural morphometric analysis of myocardium from dogs, rats, hamsters, mice, and from human hearts. Circ Res 1985, 56:377-391.

14. Boudina S, Sena S, Theobald H, Sheng X, Wright JJ, Hu XX, Aziz S, Johnson J, Bugger $H$, Zaha VG, Abel ED: Mitochondrial energetics in the heart in obesity-related diabetes: direct evidence for increased uncoupled respiration and activation of uncoupling proteins. Diabetes 2007, 56:2457-2466.

15. Boudina S, Sena S, O'Neill BT, Tathireddy P, Young ME, Abel ED: Reduced mitochondrial oxidative capacity and increased mitochondrial uncoupling impair myocardial energetics in obesity. Circulation 2005, 112:2686-2695.

16. Mansor LS, Gonzalez ER, Cole MA, Tyler DJ, Beeson JH, Clarke K, Carr CA, Heather LC: Cardiac metabolism in a new rat model of type 2 diabetes using high-fat diet with low dose streptozotocin. Cardiovasc Diabetol 2013, 12:1-1.

17. König A, Bode C, Bugger H: Diabetes mellitus and myocardial mitochondrial dysfunction: bench to bedside. Heart Fail Clin 2012, 8:551-561.

18. Shen X, Zheng S, Thongboonkerd V, Xu M, Pierce WM, Klein JB, Epstein PN: Cardiac mitochondrial damage and biogenesis in a chronic model of type 1 diabetes. Am J Physiol Endocrinol Metab 2004, 287:E896-E905.
19. Broderick TL, Haloftis G, Paulson DJ: L-propionylcarnitine enhancement of substrate oxidation and mitochondrial respiration in the diabetic rat heart. J Mol Cell Cardiol 1996, 28:331-340.

20. Larche J, Lancel S, Hassoun SM, Favory R, Decoster B, Marchetti P, Chopin C, Neviere R: Inhibition of mitochondrial permeability transition prevents sepsis-induced myocardial dysfunction and mortality. J Am Coll Cardiol 2006, 48:377-385.

21. Montgomery MK, Hallahan NL, Brown SH, Liu M, Mitchell TW, Cooney GJ, Turner N: Mouse strain-dependent variation in obesity and glucose homeostasis in response to high-fat feeding. Diabetologia 2013, 56:1129-1139.

22. Rasouli N, Kern PA: Adipocytokines and the metabolic complications of obesity. J Clin Endocrinol Metab 2008, 93:S64-S73.

23. Kern PA, Ranganathan S, Li C, Wood L, Ranganathan G: Adipose tissue tumor necrosis factor and interleukin-6 expression in human obesity and insulin resistance. Am J Physiol Endocrinol Metab 2001, 280:E745-E751.

24. Van Heek M, Compton DS, France CF, Tedesco RP, Fawzi AB, Graziano MP, Sybertz EJ, Strader CD, Davis HR: Diet-induced obese mice develop peripheral, but not central, resistance to leptin. J Clin Invest 1997, 99:385-390.

25. Coppari R, Bjørbæk C: Leptin revisited: its mechanism of action and potential for treating diabetes. Nat Rev Drug Discov 2012, 11:692-708.

26. Lago F, Gómez R, Gómez-Reino JJ, Dieguez C, Gualillo O: Adipokines as novel modulators of lipid metabolism. Trends Biochem Sci 2009, 34:500-510.

27. Hue $L$, Taegtmeyer $H$ : The randle cycle revisited: a new head for an old hat. Am J Physiol Endocrinol Metab 2009, 297:E578-E591.

28. Randle PJ, Garland PB, Hales CN, Newsholme EA: The glucose fatty-acid cycle. Its role in insulin sensitivity and the metabolic disturbances of diabetes mellitus. Lancet 1963, 1:785-789.

29. Fraulob JC, Ogg-Diamantino R, Fernandes-Santos C, Aguila MB, Mandarim-de-Lacerda CA: A mouse model of metabolic syndrome: insulin resistance, fatty liver and non-alcoholic fatty pancreas disease (NAFPD) in C57BL/6 mice fed a high fat diet. J Clin Biochem Nutr 2010, 46:212-223.

30. Ahrén J, Ahrén B, Wierup N: Increased $\beta$-cell volume in mice fed a high-fat diet: a dynamic study over 12 months. Islets 2010, 2:353-356.

31. Tabák AG, Herder C, Rathmann W, Brunner EJ, Kivimäki M: Prediabetes: a high-risk state for diabetes development. Lancet 2012, 379:2279-2290.

32. Lenzen S: The mechanisms of alloxan- and streptozotocin-induced diabetes. Diabetologia 2008, 51:216-226.

33. Reed MJ, Meszaros K, Entes $\sqcup$, Claypool MD, Pinkett JG, Gadbois TM, Reaven GM: A new rat model of type 2 diabetes: the fat-fed, streptozotocin-treated rat. Metab Clin Exp 2000, 49:1390-1394.

34. Hosokawa M, Dolci W, Thorens B: Differential sensitivity of GLUT1- and GLUT2-expressing beta cells to streptozotocin. Biochem Biophys Res Commun 2001, 289:1114-1117.

35. Trost SU, Belke DD, Bluhm WF, Meyer M, Swanson E, Dillmann WH: Overexpression of the sarcoplasmic reticulum $\mathrm{Ca}(2+)$-ATPase improves myocardial contractility in diabetic cardiomyopathy. Diabetes 2002, 51:1166-1171.

36. Kajstura J, Fiordaliso F, Andreoli AM, Li B, Chimenti S, Medow MS, Limana F, Nadal-Ginard B, Leri A, Anversa P: IGF-1 overexpression inhibits the development of diabetic cardiomyopathy and angiotensin II-mediated oxidative stress. Diabetes 2001, 50:1414-1424.

37. Lancel S, Montaigne D, Marechal X, Marciniak C, Hassoun SM, Decoster B, Ballot C, Blazejewski C, Corseaux D, Lescure B, Motterlini R, Neviere R: Carbon monoxide improves cardiac function and mitochondrial population quality in a mouse model of metabolic syndrome. PLOS One 2012, 7:e41836.

38. Marsh SA, Dell'italia $\sqcup$, Chatham JC: Interaction of diet and diabetes on cardiovascular function in rats. Am J Physiol Heart Circ Physiol 2009, 296:H282-H292.

39. Dinçer UD, Bidasee KR, Güner S, Tay A, Ozçelikay AT, Altan VM: The effect of diabetes on expression of beta1-, beta2-, and beta3-adrenoreceptors in rat hearts. Diabetes 2001, 50:455-461.

40. Boudina S, Bugger H, Sena S, O'Neill BT, Zaha VG, Ilkun O, Wright JJ, Mazumder PK, Palfreyman E, Tidwell TJ, Theobald H, Khalimonchuk O, Wayment B, Sheng X, Rodnick KJ, Centini R, Chen D, Litwin SE, Weimer BE, Abel ED: Contribution of impaired myocardial insulin signaling to mitochondrial dysfunction and oxidative stress in the heart. Circulation 2009, 119:1272-1283. 
41. Arany Z, He H, Lin J, Hoyer K, Handschin C, Toka O, Ahmad F, Matsui T, Chin S, Wu P-H, Rybkin II, Shelton JM, Manieri M, Cinti S, Schoen FJ, Bassel-Duby R, Rosenzweig A, Ingwall JS, Spiegelman BM: Transcriptional coactivator PGC-1 alpha controls the energy state and contractile function of cardiac muscle. Cell Metab 2005, 1:259-271.

42. Leone TC, Lehman JJ, Finck BN, Schaeffer PJ, Wende AR, Boudina S, Courtois M, Wozniak DF, Sambandam N, Bernal-Mizrachi C, Chen Z, Holloszy JO, Medeiros DM, Schmidt RE, Saffitz JE, Abel ED, Semenkovich CF, Kelly DP:

PGC-1alpha deficiency causes multi-system energy metabolic derangements: muscle dysfunction, abnormal weight control and hepatic steatosis. PLoS Biol 2005, 3:e101.

43. Lehman JJ, Barger PM, Kovacs A, Saffitz JE, Medeiros DM, Kelly DP: Peroxisome proliferator-activated receptor gamma coactivator-1 promotes cardiac mitochondrial biogenesis. J Clin Invest 2000, 106:847-856.

44. Xu X, Kobayashi S, Chen K, Timm D, Volden P, Huang Y, Gulick J, Yue Z, Robbins J, Epstein PN, Liang Q: Diminished autophagy limits cardiac injury in mouse models of type 1 diabetes. J Biol Chem 2013, 288:18077-18092.

45. Montaigne D, Marechal X, Coisne A, Debry N, Modine T, Fayad G, Potelle C, Arid El J-M, Mouton S, Sebti Y, Duez H, Preau S, Remy-Jouet I, Zerimech F, Koussa M, Richard V, Neviere R, Edmé JL, Lefebvre P, Staels B: Myocardial contractile dysfunction is associated with impaired mitochondrial function and dynamics in type 2 diabetic but not in obese patients. Circulation 2014, doi:10.1161/CIRCULATIONAHA.113.008476

46. Nakamura H, Matoba S, Iwai-Kanai E, Kimata M, Hoshino A, Nakaoka M, Katamura M, Okawa Y, Ariyoshi M, Mita Y, Ikeda K, Okigaki M, Adachi S, Tanaka H, Takamatsu T, Matsubara H: p53 promotes cardiac dysfunction in diabetic mellitus caused by excessive mitochondrial respiration-mediated reactive oxygen species generation and lipid accumulation. Circ Heart Fail 2012, 5:106-115.

47. Lashin O, Romani A: Hyperglycemia does not alter state 3 respiration in cardiac mitochondria from type-I diabetic rats. Mol Cell Biochem 2004, 267:31-37.

48. Bugger $H$, Riehle C, Jaishy B, Wende AR, Tuinei J, Chen D, Soto J, Pires KM, Boudina S, Theobald HA, Luptak I, Wayment B, Wang X, Litwin SE, Weimer BC, Abel ED: Genetic loss of insulin receptors worsens cardiac efficiency in diabetes. J Mol Cell Cardiol 2012, 52:1019-1026.

49. Vadvalkar SS, Baily CN, Matsuzaki S, West M, Tesiram YA, Humphries KM: Metabolic inflexibility and protein lysine acetylation in heart mitochondria of a chronic model of type 1 diabetes. Biochem $J$ 2013, 449:253-261.

50. Fancher IS, Dick GM, Hollander JM: Diabetes mellitus reduces the function and expression of ATP-dependent $\mathrm{K}+$ channels in cardiac mitochondria. Life Sci 2013, 92:664-668.

doi:10.1186/s12933-014-0118-7

Cite this article as: Marciniak et al:: Cardiac contractile function and mitochondrial respiration in diabetes-related mouse models.

Cardiovascular Diabetology 2014 13:118.

\section{Submit your next manuscript to BioMed Central and take full advantage of:}

- Convenient online submission

- Thorough peer review

- No space constraints or color figure charges

- Immediate publication on acceptance

- Inclusion in PubMed, CAS, Scopus and Google Scholar

- Research which is freely available for redistribution 